\title{
1. SURVEY OF SPIRAL STRUCTURE PROBLEMS
}

\author{
J. H. OORT \\ Leiden Observatory, Leiden, The Netherlands
}

If one tries to look at the structure of spiral galaxies with the eyes of an unbiased newcomer, and if one then pages through the Hubble Atlas of Galaxies or Arp's Atlas of Peculiar Galaxies the first reaction is that these objects must be young, and could hardly have lived over much more than one revolution. Consideration of data on the strong differential rotation that is characteristic for spiral galaxies further strengthens this impression.

And yet we know, from convincing age determinations of clusters, that at least our own Galaxy is old. There is, furthermore, so much similarity between the spectral characteristics and general composition of other spirals and so much resemblance with our Galaxy that it seems unreasonable to believe that these other spirals would be much younger. We may assume, therefore, that the ages of most spiral galaxies count several dozens of revolutions. Let us adopt 50 revolutions as an order-ofmagnitude for the average age.

These high ages are for the systems as a whole. They do not necessarily apply to the spiral patterns, which might be more ephemeral. But if we would suppose the spiral arms to be short-lived there has to be a mechanism by which they are revived every few revolutions. Otherwise we could not explain why at present the majority of non-elliptical stellar systems show a well-defined arm structure. A particular difficulty is that they would have to be revived as more or less continuous structures throughout the entire system. Up to the present no plausible mechanism has been worked out which could explain such a periodical renewal of the large-scale spiral structures.*

It was suggested many years ago by Bertil Lindblad that spiral patterns could be maintained for a long period by a sectorial wave system. This idea has in recent years been further worked out by Lin, Shu and Yuan, who showed among other things how such a density wave causing a spiral pattern could be sustained by its own spiral gravitational field superposed on the general axisymmetrical field of the galaxy. The density excess in the arms needed for this spiral field is entirely reasonable in view of the observational data on the distribution of gas and young stars in the Galaxy. As will be indicated in some reports at the Symposium there is considerable direct evidence, especially in the humps of the rotation curve found from $21-\mathrm{cm}$ observations in our Galaxy, but likewise in other $21-\mathrm{cm}$ observations, that streamings of the kind and amplitude required by the wave theory, actually exist. Moreover there is evidence that formation of stars occurs preferentially when the gas in the regions concerned is compressed by the wave.

* In the course of the Symposium this problem was extensively discussed. It appeared that several promising theories on the re-excitation of spiral structure had already been partly worked out. 
A very important step forward thus appears to have been made in understanding how spiral patterns can persist for a much longer time than the particular arms we see at a given instant.

The theory explains the maintenance but not the origin of spiral structure. I do not think this is an important shortcoming, for it is easy to conceive of processes which would start a spiral structure. Indeed, any mechanism of formation of a rotating galaxy is likely to produce a system with large scale initial asymmetry in the plane of rotation, which will almost automatically develop into a two-armed spiral structure.

A more serious problem seems that of the long-term permanence of the spiral waves. Can they continue to run round during 50 revolutions without fatal damage to their regularity? Looking at the irregularities in the actual spiral galaxies one wonders whether the present spirals could continue to exist for such a large number of revolutions. The problem seems particularly acute for the outer parts of Sc spirals, like M 101 or NGC 628, and still more for some SBc systems, like NGC 3187 in the Leo group. In the latter case interaction with other members of the group might possibly have been a factor in determining the non-equilibrium shape of arms. But in most cases we cannot invoke interaction. Moreover, if there had been interaction in the past it might well have contributed to disturb the regular wave pattern, but less likely to rebuild it.

The foregoing considerations give one the feeling that, though the principal part of the spiral phenomenon may be explained by wave motions of the type described, there is something else besides which influences, or perhaps incites, the structure.

I have not yet mentioned the important class of spirals which beside spiral arms have a bar structure. The difficult dynamical problems which these systems present will be reported on by Freeman. The wave theory cannot be directly applied to barred spirals. Interesting observations of velocity patterns made by Mrs. Burbidge indicate that the phenomena are in any case quite complicated.

There are, of course, also the irregular galaxies to be considered. In addition, some rather regular galaxies exist whose forms do not fit in any of the categories considered (cf. the ring galaxy reproduced as No. 147 in Arp's Atlas of Peculiar Galaxies).

At this point I want to say a few words about the general appearance of spiral galaxies. Dr. Lin has sometime quoted me as having stated that the important characteristic of spiral galaxies is not that they have spiral-like features - because spiral shapes are the natural shape into which any larger feature will automatically be drawn out by differential rotation - but that in so many cases spiral arms can be followed more or less continuously through the entire galaxy. I do not want to withdraw this statement, but I must point out that it should be supplemented by two essential additions. First, that in about half of the spirals the structure is either unclear, or there are more than two arms. Second, that even in the half that can be classed among the two-armed spirals there are invariably important additional features between the two principal arms, while the latter have often a number of secondary branches coming off their outer rims. In a fair number of cases these secondary features make it difficult to follow the main arms throughout the system. 
What is already difficult in extraneous systems is an order of magnitude more difficult in the galaxy which is the title subject of this symposium. Our own Galaxy is seen edge-on, and almost the only way to unravel the general structure is through observation of radial velocities. But these are affected as much as the structures themselves by the irregularities described, so that we get two classes of irregularities superimposed. Nevertheless it has proved possible by means of the $21-\mathrm{cm}$ observations, recently aided also by velocities of $\mathrm{H}$ II regions derived from radio recombination lines, to trace spiral arms through at least one half of the Galactic System. A full discussion of this will be given in the course of this symposium by Kerr, Mezger, Westerhout, Weaver, Varsavsky, and, for the inner arms, by Burton and Shane.

Though for a general study of the spiral structure the Galactic System is less accessible than other galaxies it does permit the study of a number of phenomena connected with the spiral arms which, so far, it has not been possible to investigate in other galaxies. I mention the detailed study of the stellar populations of spiral arms, the distribution of individual motions of stars in the arms, the thickness of the arms perpendicular to the galactic plane, the connection between the arms and large-scale streamings of the gas, the character of large-scale irregularities like the splitting of the Perseus arm discovered by Münch and extensively studied by Rickard, and, particularly, the large radial motions of the arms in the central region.

As the latter will not be discussed in other parts of the symposium, except in connection with the Scutum arm, I want to say a few words about the possible significance of these phenomena.

In the region within 3 or $4 \mathrm{kpc}$ from the centre we find an arm which at the point where it passes between the centre and us has a velocity component of $53 \mathrm{~km} \mathrm{~s}^{-1}$ directed away from the centre. This so-called 3-kpc arm has all the characteristics of the spiral arms observed in the outer parts of the Galaxy except for its large radial motion. It contains a similar order of mass per unit length, has a similar linear thickness, and is a major feature, that can be followed over at least a full quadrant of galactocentric longitude. On the far side of the nucleus there is an approximately equal amount of hydrogen, apparently also moving away from the centre, but with velocities which are two to three times higher. Part of this matter may be rather close to the centre. The arm structures on that side are not so well-defined as the $3-\mathrm{kpc}$ arm.

The problem how these expanding arms have acquired their outward motions is still unsolved. It is tempting to think, as several astronomers have done, that the outward momentum comes from violent ejections from the galactic nucleus. A realistic basis for this idea has been furnished by recent detailed investigations of Seyfert nuclei, showing that violent motions involving large and massive individual clouds exist in the nuclei of these otherwise normal spirals. Clouds or streams with masses of the order of $10^{4}$ or $10^{5}$ solar masses are apparently escaping from their nuclei at velocities of the order of $1000 \mathrm{~km} \mathrm{~s}^{-1}$. If a comparable activity had happened in our Galaxy it might well have caused the observed outward motions of the arms in the central region. It is interesting that rather direct evidence of activity of a similar kind has now been found in the vicinity of the nucleus of the Galactic System. But 
the velocities are an order of a magnitude lower than in the Seyfert nuclei. The evidence for the ejection of large masses of gas from the nuclear region was first discovered in 1966 by W. W. Shane from a survey for high-velocity features in the central region outside the galactic plane. Van der Kruit, who has followed up this discovery, finds that within a region extending to $5^{\circ}$ in latitude and $10^{\circ}$ in longitude from the centre there exists a mass of roughly $5 \times 10^{6} M_{\odot}$ of neutral hydrogen well outside the galactic disk. His observations suggest an ejection of clouds from the nucleus in two roughly opposite directions making large angles with the plane. The velocities range from about $100 \mathrm{~km} \mathrm{~s}^{-1}$ near the centre to $50 \mathrm{~km} \mathrm{~s}^{-1}$ at 5 to $10^{\circ}$ distance. Though the momentum involved is insufficient to have yielded the motion of the 3-kpc arm, the observed part of which has a mass of $4 \times 10^{7} M_{\odot}$, we might imagine that strong nuclear activity is a thing that comes in bursts, that we are now in a relatively quiescent period, and that the motion of the 3-kpc arm is due to much stronger activity at an epoch a few times $10^{7}$ years ago. The circumstance that the streams appear to be ejected at a considerable angle with the galactic plane makes it understandable that the small rotating nuclear disk found by Rougoor and Oort might have survived the period of violent activity.

The preferential direction might well be connected with the structure of the magnetic field. It is suggestive, in this connection, that Kerr and Sinclair have found that the emission at $20 \mathrm{~cm}$ near the centre shows two separate ridges, one practically along the galactic circle, the other along a line going through the centre but inclined at an angle of $47^{\circ}$ to this circle. The latter ridges not only lie in the same quadrants as the high-velocity streams, but also the inclination to the galactic equator is comparable to what the streams indicate in the region close to the centre. The inclined radiation ridge has not been followed for more than 1.4 from the centre. Kerr and Sinclair suggest that it is due to jets from the nucleus. No data have yet been published on the spectrum of this most interesting radiation.

If the motion of the 3-kpc arm is ascribed to ejections from the nucleus one should ask also what consequences such spasmodic massive violence would have on the more outlying parts of the disk. Could this possibly enhance the spiral waves in these outer parts, and thereby keep them going for longer periods than if left entirely to themselves?

In this connection a special interest attaches to the observations of the Scutum arm, which Shane will discuss. The observations are indicative of an expanding motion also in this arm. Likewise in this connection I must mention the extremely interesting observations by Mrs Rubin and Ford on the central region of M 31. The almost zero velocity of rotation which they find for the gas at $2 \mathrm{kpc}$ from the centre may well have been caused by matter with little or no angular momentum expelled from the nucleus under an angle with the galactic plane, and having again collected in the plane around a distance of $2 \mathrm{kpc}$, leaving the rapidly rotating gas which is observed inside this distance more or less undisturbed.

We are clearly not yet in a position to answer the question of a possible excitation of spiral structure by the nuclear activity - at any rate not in a positive sense. The idea 
itself that the entire structure of a galaxy is strongly influenced by phenomena originating in its nucleus has been expressed on several occasions by Ambartsumian. It has very recently been suggested anew by Arp.

There are two more points I want to mention before concluding this survey.

The first is the extension of the arms perpendicular to the plane. Their average thickness is about 250 parsecs. But they have long 'wings' which, at least for the outer arms, extend to distances of 1 to $2 \mathrm{kpc}$ from the plane. These phenomena will be discussed in a later communication. A remarkable thing about these wings is that they have no tendency to merge into a more or less continuous halo, but seem to have remained connected with the arms from which they have originated. They must be highly unstable, and, consequently, should be continually renewed. An important question to be answered - also in connection with the general problem of the spiral arms - is how this repopulation of the very high $z$-levels is done, whether by violent events, or by some kind of magnetic field instability.

The second and last point is the influence on the dynamics of spiral arms of a possible inflow of gas from intergalactic space. There is, in my opinion, strong evidence that gas is flowing into the Galaxy from outside. This subject will not be discussed in the present conference. It is useful, however, to give a few numbers indicating the magnitude of the interaction with the disk gas as inferred from the high- and intermediate-velocity clouds observed in Groningen and Leiden. From these I have estimated a flow into the Galaxy of roughly $3 \times 10^{17}$ atoms per $\mathrm{cm}^{2}$ of the galactic plane per $10^{6}$ years. This is a considerable amount. In one revolution of the Galaxy it would increase the amount of gas in the disk by roughly $10 \%$, and might sensibly decrease the rotational velocity of this gas. 